
\title{
28 Research Square \\ Co-Existing Serous Epithelial Ovarian cancer with Hepatocellular Carcinoma: A Rare Case Report
}

Haote Jiang

Wenzhou Medical University

Xiaowan Huang

The First Affiliated Hospital of Wenzhou Medical University

Jianmin Li

The First Affiliated Hospital of Wenzhou Medical University

\section{Yunfeng Shan}

The First Affiliated Hospital of Wenzhou Medical University

Jinglin Xia

The First Affiliated Hospital of Wenzhou Medical University

Yuyang Zhang ( $\square$ zhangyuyang@wmu.edu.cn )

The First Affiliated Hospital of Wenzhou Medical University https://orcid.org/0000-0002-6524-8133

\section{Case Report}

Keywords: multiple primary cancers (MPCs), ovarian cancer, liver cancer, case report

Posted Date: December 11th, 2020

DOl: https://doi.org/10.21203/rs.3.rs-124599/v1

License: (c) (i) This work is licensed under a Creative Commons Attribution 4.0 International License.

Read Full License 


\section{Abstract}

\section{Background}

Serous epithelial ovarian cancer co-existing with primary liver cancer is a rare situation, and only few cases have been reported that patients have multiple primary cancers (MPCs) with ovarian cancer.

\section{Case presentation}

We present the case of a 57-year-old patient whose imaging examination results suggested liver and ovarian lesions. The patients underwent a positron emission computed tomography that revealed the presence of hypermetabolic areas in bilateral adnexal, uterus and pelvic region. After discussion at the multidisciplinary team meeting, the patient underwent surgery and her pathological results were highgrade serous epithelial ovarian cancer and hepatocellular carcinoma (mixed type), respectively. Complete cytoreduction was successfully performed. Her elevated tumor markers like AFP and CA125 decreased immediately after the operation. Now she has received 4 cycles of chemotherapy. The patient showed good tolerance, and showed no sign of recurrence.

\section{Conclusion}

The present study delineates a rarely reported case of disease with a relatively high incidence rate. Therefore, further studies are required for the perfection of its diagnosis, prevention, and treatment. This case could be a reference for the diagnosis and treatment of ovarian cancer with metastasis of ovarian cancer and other primary tumor.

\section{Background}

When ovarian cancer and liver cancer both occur at the same time, it would usually be advanced ovarian cancer with hepatic metastases. Some advanced cancers with distant metastases are often confused with multiple primary cancers (MPCs).

A retrospective study from 2002 to 2010 revealed that among 164211 female cadavers, 268 women had been found to have multiple primary cancers(MPCs) with ovarian cancer; however, no cases of primary ovarian cancer complicated with liver cancer were found[1]. In an epidemiological review, the amount of MPCs over all cancer sites in the literature is reported to be in the range between 2-17\%[2].

We are going to present a case about diagnosis and treatment of a patient with ovarian cancer complicated with liver cancer.

\section{Case Presentation}

A 57-year-old woman complained about stabbing pain in lower abdominal for at least 1 month. She then went to the local hospital and the Second Affiliated Hospital of Wenzhou Medical University, respectively. 
Her CT results showed that the bilateral adnexal area was cystic, and the lateral segment of the left liver was occupied. Her blood test of tumor markers showed: CA125:564.1 ng/ml, AFP:55923 ng/ml.

Seeking for surgical treatment,she came to the Department of Hepatobiliary Surgery of our hospital on April 10, 2020. The patient had no primary comorbidities, genetic or environmental exposure to risk factors, and with no family history of cancer. Her ultrasound suggested pelvic masses in bilateral adnexal(malignant tumor first considered $\square$ right mass measured $60 * 47 * 55 \mathrm{~mm} \square$ left mass measured $42 \star 33 * 34 \mathrm{~mm}$ )and peritoneal metastasis. Her first diagnosis were ovarian tumor(?), liver tumoe,Ovarian cancer liver metastasis(?) and Ovarian cancer peritoneal metastasis(?). In order to distinguish a benign liver tumor from a malignant one $\llbracket$ a liver biopsy was conducted. Before the result of biopsy reported, metastatic liver tumor of ovary was first considered. So patient was transferred to the Gynecology department. During her bimanual examination ,we found a palpable mass (measured $6 \star 5 \star 5 \mathrm{~cm}$ ) with pressing pain at right adnexal region and another mass (measured $4 \star 3 \star 3 \mathrm{~cm}$ ) at left adnexal region. Her enhanced abdominal computer tomography examination showed: a space-occupying lesion in left lobular liver (measured 73*100 mm) and multiple cystic-solid pelvic masses with internal segmentation (considered metastases originated from adnexal with uneven enhanced echoes and measured $77 \star 41 \mathrm{~mm}$ ). (Fig. 1.) Then her biopsy result showed the pathological type of her liver lesion was hepatocellular cancer.

After discussion at the multidisciplinary team meeting, the patient underwent surgery. The operation composed of total hysterectomy, bilateral salpingo-oophorectomy, omentectomy, partial hepatectomy, excision of lesion of bladder, mesenteric and peritoneum, repair of diaphragm, and bilateral ureteral stent implantation. During surgery, we found a $7 * 7 * 6 \mathrm{~cm}$ solid-cystic mass in right adnexal and peritoneal adhesion. The left adnexal was wrapped by peritoneal and adhered to posterior of uterus. There were two hard masses measured about $3 * 3 \mathrm{~cm}$ on the surface of the bladder and the right peritoneal. The left lateral lobe of liver showed a 10-cm-sized mass, involving the diaphragm. No suspicious or enlarged lymph node was found.

After surgery, the result of cytology examination was negative for malignancy cell( AFP:8345 ng/ml, CA125:333.4 ng/ml) immediately after the operation. Her latest data of biomarkers were examed in 2020.7.13 and showed at the normal level(AFP:4.57 ng/ml; CA125:27.6 U/ml). Combined with the results of immunohistochemistry(Figure 2), the masses on the surface of the greater omentum, mesentery and bladder were diagnosed high-grade serous ovarian cancer metastasis. According to the pathology and immunohistochemistry results, the patient's diagnosis was serous ovarian cancer FIGO stage

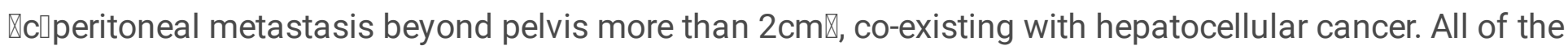
lesions from both liver and ovary were resected completely. After consultation from hepatobiliary department, it was considered that no follow-up treatment for hepatocellular cancer was needed. According to National Comprehensive Cancer Network (NCCN)Clinical Practice Guidelines of Ovarian Cancer(version 1.2020), she would receive 6 cycles of combined chemotherapy of paclitaxel $(240 \mathrm{mg})$ plus carboplatin. 


\section{Discussion And Conclusion}

MPCs means that tumors have been found in one or multiple organs in one patient at same time or at different period. The incidence of multiple primary cancer is much lower than that of primary tumor metastasis and usually may be misdiagnosed.

With the widespread use of sensitive and systemic imaging techniques, the early detection rate of metastases or MPCs has increased. In recent years, The incidence of multiple primary cancers has gradually increased and has usually been found in the neck and head, but it is rarely reported in the female genital system.

In more than half of the reported cases, the MPCs are confined to the same organ or to bilaterally paired organs. The etiology of MPCs is still not clear, but some risk factors are widely accepted such as adverse treatment reaction of radiotherapy or chemotherapy, environmental factors (long-term exposure to radiation pollution), lifestyle (alcoholic and tobacco use) and genetic susceptibility[3].

The median process free survival (PFS) and overall survival (OS) for this group of stage IV ovarian cancer patients was 12 and 29 months, respectively[4]. Because it is hard for patients with advanced ovarian cancer to have an optimal cytoreduction or be able to undergo primary cytoreduction. If we could confirm the liver lesion does not originate from the ovarian cancer, the cancer stage can be reduced. Immunohistochemistry is the most important tool for the distinguishing diagnostic.

The treatment for patients with synchronous multiple primaries is challenging. Doctors need to balance both drug-efficiency and drug-related toxicity because of the different chemotherapy regimens for each primary cancer and to figure out what is the most critical tumor in terms of prognosis. The task to establish a general treatment guideline for all situations of MPCs renders a strenuous process due to the varying combinations of MPCs and small number of individual cases being currently identified. It is not a simple addition of each primary cancer treatment regimen, but an individualized management through a multidisciplinary approach.

According to Global surveillance of trends in cancer survival 2000-12(CONCORD-3,the five-year survival rate of liver cancer and ovarian cancer are $14.1 \%$ and $41.8 \%$ respectively[5]. With no more similar cases to refer to, it is difficult to judge the prognosis of this patient. The further prognostic would be monitored by regular follow-up plan(visits every 2-4 month for 2 years,then 3-6 month for 3 years $\square$ then annually after 5 years). The follow-up plan should be continued. If there were signs that showed relapses, the doctors need to consider whether histological types of recurrent lesion are derived from ovary or liver in order to formulate the next step of treatment. The patient was reviewed in the outpatient clinic on time after the whole therapy. Now she have finished her chemotherapy and her latest PET-CT result showed no recurrence.

This is an extremely rare case of high-grade serous ovarian cancer co-existing with hepatic cell cancer. The diagnosis and treatment of this patient can be used as a reference for similar cases. With the 
incidence of multiple primary cancer increased, different cases should be shared and to summarize a reliable treatment guide.

\section{Abbreviations}

MPCs: multiple primary cancers; AFP:alpha fetoprotein

\section{Declarations}

\section{PATIENT PERSPECTIVE}

The patient hopes that the lesion can be completely removed as much as possible, and the pain of subsequent treatment can be reduced. She had no surgical complications and no obvious side effects during subsequent chemotherapy.

\section{PATIENT'S CONSENT}

Patient has provided informed consent for publication of the case

\section{CONFLICT OF INTERESTS}

The authors declare that there are no conflict of interests.

\section{DATA AVAILABILITY STATEMENT}

Data sharing is not applicable to this article as no new data were created or analysed in this study.

\section{References}

1. Miki, Y., et al., Multiple primary cancers associated with endometrial and ovarian cancers: An analysis based upon the Japan Autopsy Annual Database from 2002 to 2010. J Obstet Gynaecol Res, 2019. 45(5): p. 1012-1018.

2. Vogt, A., et al., Multiple primary tumours: challenges and approaches, a review. ESMO Open, 2017. 2(2): p. e000172.

3. LB, T., et al., Cancer survivorship-genetic susceptibility and second primary cancers: research strategies and recommendations. Journal of the National Cancer Institute, 2006. 98(1): p. 15-25

4. WE, W., et al., Tumor residual after surgical cytoreduction in prediction of clinical outcome in stage IV epithelial ovarian cancer: a Gynecologic Oncology Group Study. Journal of clinical oncology : official journal of the American Society of Clinical Oncology, 2008. 26(1): p. 83-9

5. Allemani, C., et al., Global surveillance of trends in cancer survival 2000-14 (CONCORD-3): analysis of individual records for 37513025 patients diagnosed with one of 18 cancers from 322 populationbased registries in 71 countries. Lancet, 2018. 391(10125): p. 1023-1075 


\section{Figures}
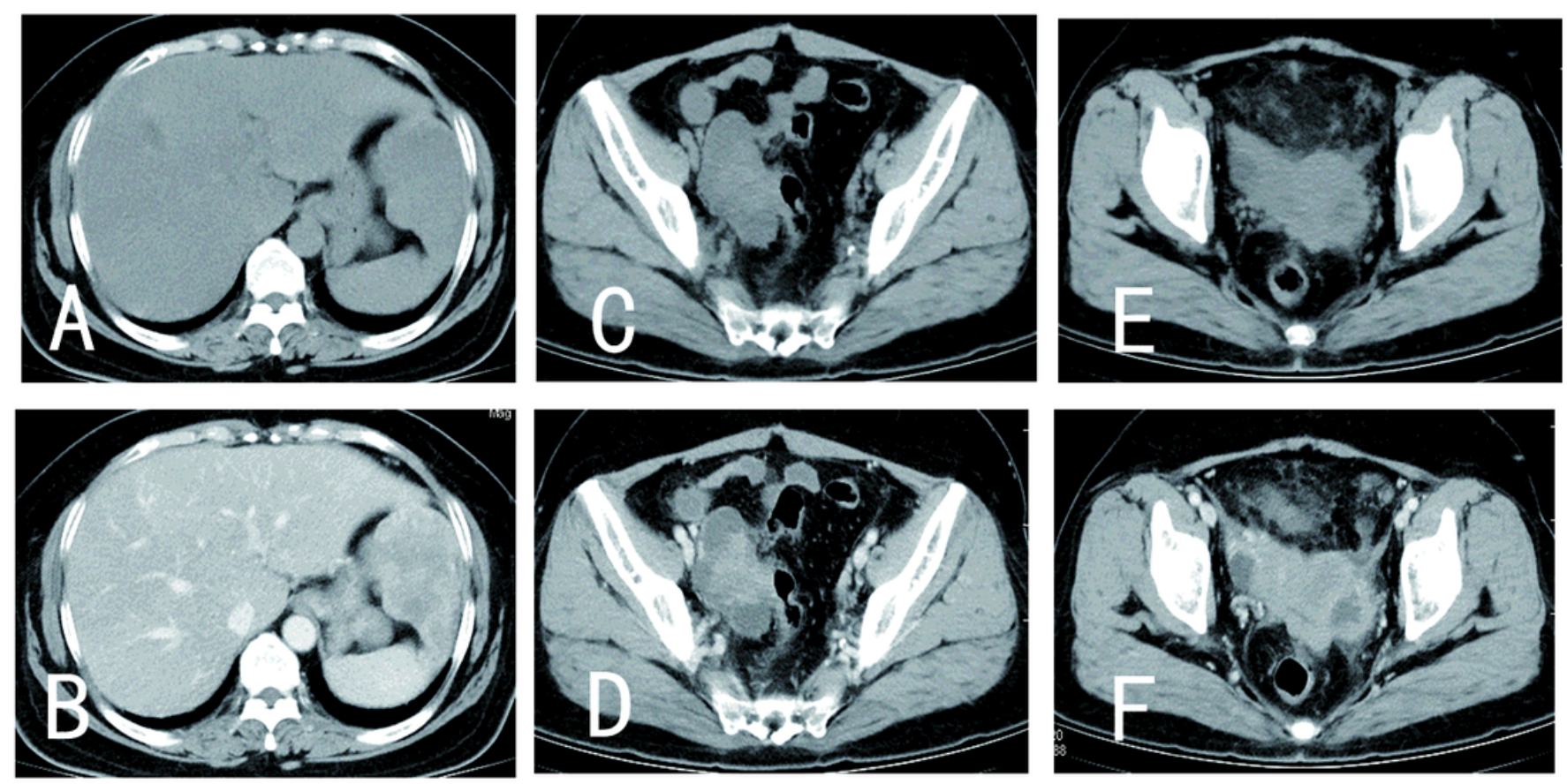

Figure 1

A. Abdominal CT scan demonstrates a soft tissue density mass at the left lateral lobe of liver. B. Contrastenhanced CT scans shows the liver lesion significant heterogenous enhancement. C口E. Abdominal CT scan shows that soft tissue density masses with cystic and solid components in bilateral adnexal. DaF. Contrast-enhanced CT scans shows the solid component of both side of lesions was markedly enhanced 

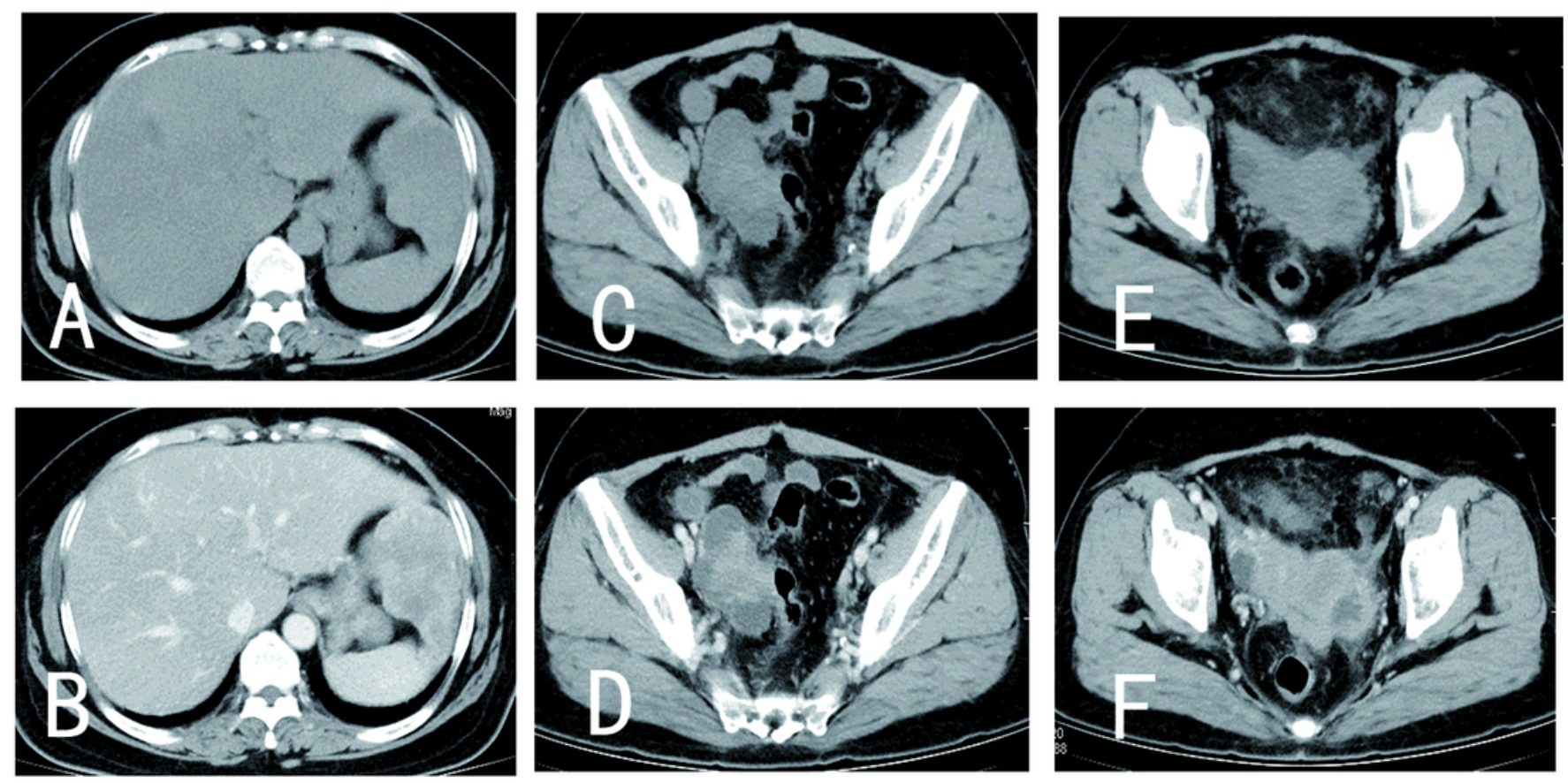

Figure 1

A. Abdominal CT scan demonstrates a soft tissue density mass at the left lateral lobe of liver. B. Contrastenhanced CT scans shows the liver lesion significant heterogenous enhancement. C口E. Abdominal CT scan shows that soft tissue density masses with cystic and solid components in bilateral adnexal. DDF. Contrast-enhanced CT scans shows the solid component of both side of lesions was markedly enhanced 

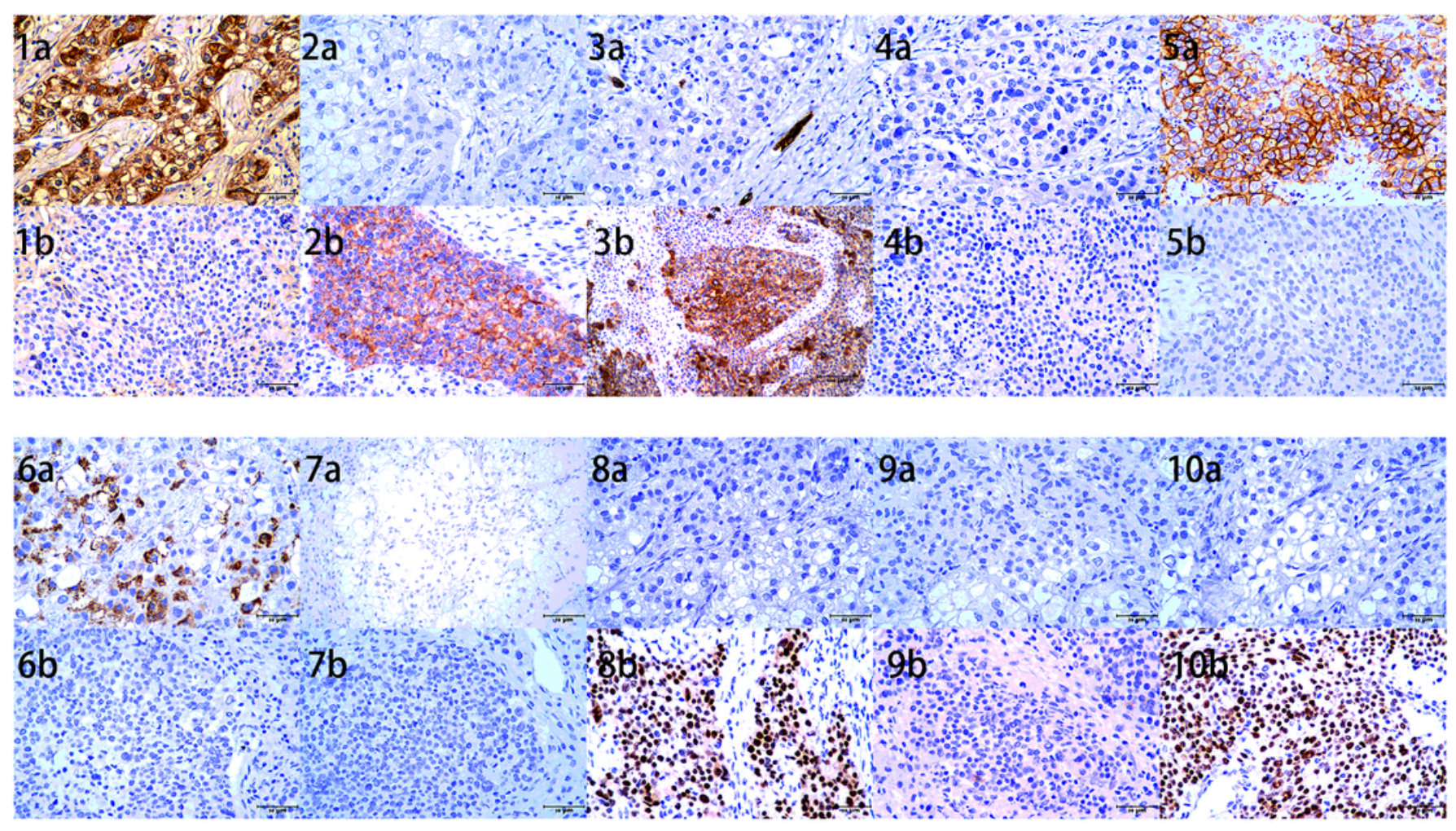

Figure 2

Immunohistochemistry staining result ( a囚ovarian mass खb冈liver mass )( 1:AFP; 2:CA125; 3: CK7; 4: CK20; 5: GPC-3; 6:Hepatocyte; 7:OCT-34; 8:PAX8; 9:PLAP; 10:WT-1) 

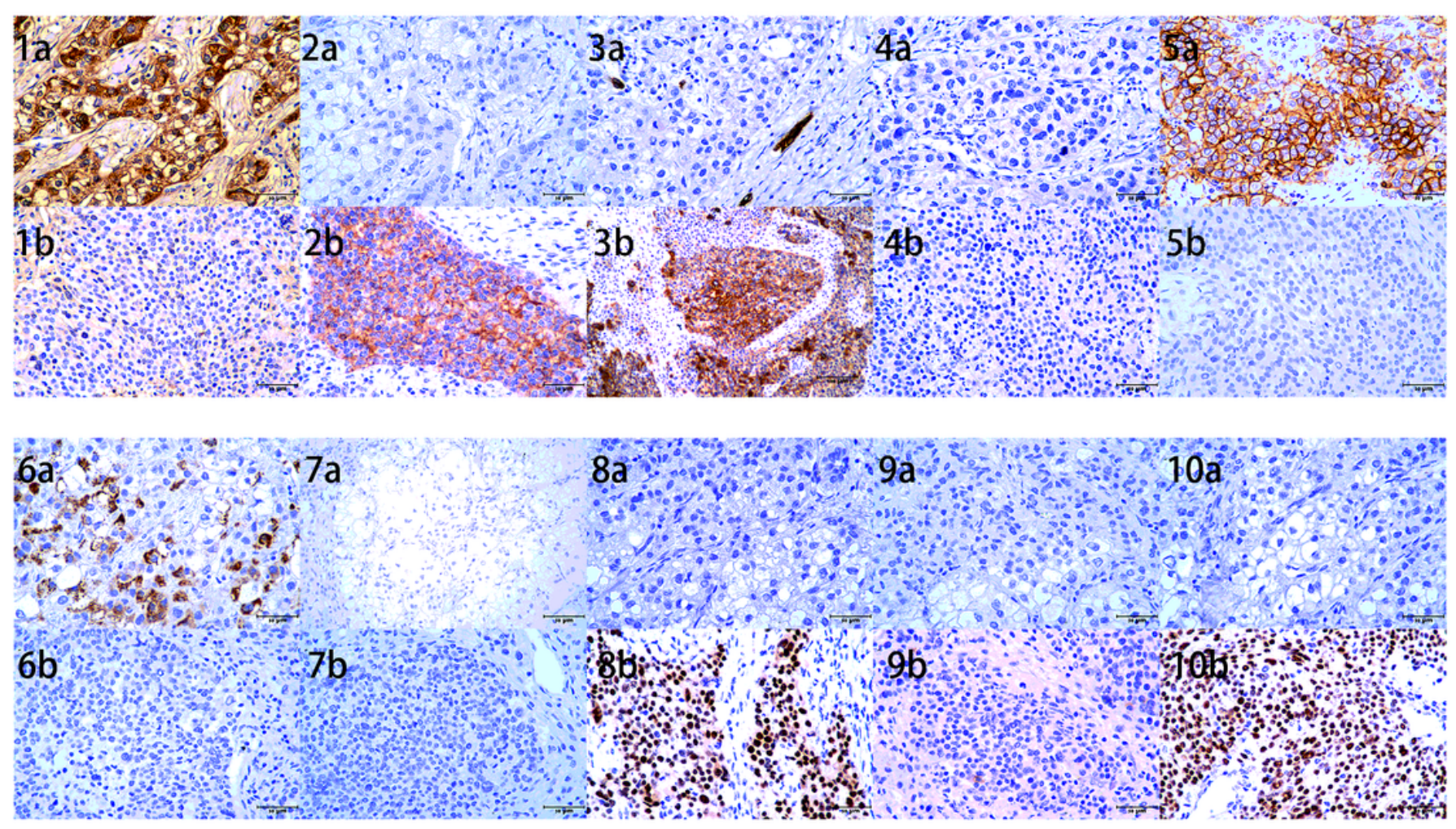

Figure 2

Immunohistochemistry staining result ( a囚ovarian mass खb冈liver mass )( 1:AFP; 2:CA125; 3: CK7; 4: CK20; 5: GPC-3; 6:Hepatocyte; 7:OCT-34; 8:PAX8; 9:PLAP; 10:WT-1)

\section{Supplementary Files}

This is a list of supplementary files associated with this preprint. Click to download.

- carelist1.pdf

- carelist1.pdf 